\title{
Kernel-based Logical and Relational Learning with kLog for Hedge Cue Detection
}

\author{
Mathias Verbeke ${ }^{1}$, Paolo Frasconi ${ }^{2}$, Vincent Van Asch ${ }^{3}$, Roser Morante ${ }^{3}$, \\ Walter Daelemans ${ }^{3}$, and Luc De Raedt ${ }^{1}$ \\ 1 Department of Computer Science, Katholieke Universiteit Leuven, Belgium \\ 2 Dipartimento di Sistemi e Informatica, Università degli Studi di Firenze \\ 3 Department of Linguistics, Universiteit Antwerpen, Belgium
}

\begin{abstract}
Hedge cue detection is a Natural Language Processing (NLP) task that consists of determining whether sentences contain hedges. These linguistic devices indicate that authors do not or cannot back up their opinions or statements with facts. This binary classification problem, i.e. distinguishing factual versus uncertain sentences, only recently received attention in the NLP community. We use kLog, a new logical and relational language for kernel-based learning, to tackle this problem. We present results on the CoNLL 2010 benchmark dataset that consists of a set of paragraphs from Wikipedia, one of the domains in which uncertainty detection has become important. Our approach shows competitive results compared to state-of-the-art systems.
\end{abstract}

